M. Aragüés-Peñalba, J. Rimez, J. Beerten, D. Van Hertem, O. Gomis-Bellmunt, "Secure and optimal operation of hybrid AC/DC grids with large penetration of offshore wind," Proc. IET International Conference on AC and DC Power Transmission ACDC 2015, 11th ed., Birmingham, UK, Feb. 10-12, 2015, 9 pages.

Digital Object Identifier: http://dx.doi.org/10.1049/cp.2015.0034

URL (IET Digital Library):

http://digital-library.theiet.org/content/conferences/10.1049/cp.2015.0034

URL (IEEE Xplore Digital Library):

http://ieeexplore.ieee.org/xpl/articleDetails.jsp?arnumber=7140517

(C) 2015 IET. This paper is a postprint of a paper submitted to and accepted for publication in Proc. IET International Conference on AC and DC Power Transmission 2015 and is subject to Institution of Engineering and Technology Copyright. The copy of record is available at IET Digital Library. 


\title{
Secure and optimal operation of hybrid AC/DC grids with large penetration of offshore wind
}

\author{
Mònica Aragüiés-Peñalba*, Johan Rimez ${ }^{* *}$, Jef Beerten ${ }^{\dagger}$, Dirk Van Hertem ${ }^{\dagger}$, Oriol Gomis-Bellmunt* \\ * CITCEA-UPC, Departament d'Enginyeria Elèctrica, Universitat Politècnica de Catalunya, Barcelona, Spain, \\ $\dagger$ ELECTA, Electrical Engineering Department, KU Leuven, Leuven, Belgium, \\ ** Elia system operator, Brussels, Belgium
}

Keywords: Optimal Power Flow, HVDC, HVAC, Reliability, $\mathrm{N}-1$ vs N-0

\begin{abstract}
This study focuses on the the reliability of HVDC grids, analysing the secure and optimal operation of transmission systems consisting of both HVDC (High Voltage Direct Current) and HVAC (High Voltage Alternating Current) interconnections, with high penetration of offshore wind, taking into account the system spinning reserves. While AC systems are usually operated N-1, it is not clear which should be the criteria for operating DC grids. The consequences of operating a DC grid with different levels of security are addressed and the expected cost of operation and wind power curtailment over one year are determined, taking into account the probability of failure of the different power system components. A study case, based on the CIGRÉ HVDC Test System, is presented, showing the system response to the outage of different elements and its effect on the cost of operation. Generic wind profiles and representative AC generation parks are used to model the system behaviour over a complete year.
\end{abstract}

\section{Introduction}

The security of a power system represents the degree of risk in its ability to overcome disturbances (contingencies) without interruption of customer services [1]. Contingencies refer to outages such as sudden and not scheduled loss of service of one or more of the main power system components [1]. Normally, power systems are operated according to the deterministic $\mathrm{N}-1$ criterion, so the permanent loss of one power system component may not affect the stable operation of the rest of the system. However, recent changes in power systems are influencing the way they are planned, operated and controlled.

As detailed in [2], power systems have to deal with an increasing demand, with not always an appropriate adaptation of generation and transmission, leading to more stressed operating conditions. On the other hand, the larger penetration of renewable energy generation implies larger uncertainty in systems operation. For instance, in a system with large penetration of offshore wind, the uncertainty and fluctuating behaviour inherent to this power source can compromise its secure operation, as the wind power is not always available when needed to react to an outage, or can worsen the situation in case the wind power cannot be absorbed or when the grid is not able to accommodate the power flows. Due to these aspects, the security of power systems is being compromised, endangering the accomplishment of the N-1 criterion in some cases. In addition, new devices such as HVDC or FACTS, are being installed, enabling renewable power integration through advanced controls, but at the same time adding complexity to the power system control.

The European transmission system is being extended by combining both AC and DC technologies. With the projected advent of HVDC grids, contingencies such as converter outages cause a quasi-instantaneous power redistribution by means of a voltage droop control [3], in a way that shows similarities with the frequency droop control in the AC system. However, these power redistributions might result in line overloads when not taken into account properly. All this adds to the complexity of the study of the reliability of such systems, as it is not yet clear to which level of reliability hybrid HVAC-HVDC systems should be operated.

Some authors have analysed the optimal operation of AC grids and DC grids [4-7]. Among these studies, the first one that defines and optimizes, for different objective functions, DC and AC load flows simultaneously in a random AC-DC network, allowing the possibility of meshing the DC system is [7]. The tool developed in [7] is therefore applied in the present study to a scenario with large penetration of offshore wind.

Some studies regarding SCOPF (Security Constrained Optimal Power Flow) that takes into account wind power integration exist: [8-11]. The authors from [8] propose a SCOPF to allow the post-contingency control of a VSC-HVDC system through corrective actions based on the current injection method. But the only contingency analysed is the outage of DC lines. In [9] a probabilistic robust generation dispatch to guarantee $\mathrm{N}-1$ secure under wind uncertainty, is proposed, but it is assumed that enough reserves are available to balance the generation-load mismatch, so no reserve scheduling is considered. This analysis is extended to multi-area systems involving different levels of data exchange in [11]. In [10], the authors from [9, 11] design a N-1 secure day-ahead dispatch, while determining 
the minimum cost reserves for power systems with high wind penetration. So, new constraints ensure that the scheduled generation dispatch plus the reserve contribution are inside the generation capacity limits. Here, ramping up and down reserves are considered. Although the studies from $[9,11]$ are detailed, they only focus on the AC system. A scenario with HVDC links is not contemplated.

This study analyses the secure and optimal operation of hybrid HVAC-HVDC connected systems with large penetration of offshore wind, taking into consideration the system spinning reserves. The operation and economic consequences of requiring higher or lower security are investigated. Using the SCOPF tool for hybrid AC/DC power systems from [7], it is possible to optimize a specified objective function while guaranteeing all the equality and inequality constraints limiting the electrical variables. Security constraints are included and both preventive and corrective actions of the TSO (Transmission System Operator) can be used to ensure security. Several different objective functions can be defined.

\section{Optimal operation of hybrid AC/DC systems including spinning reserves}

\subsection{SCOPF tool}

The optimal operation of hybrid AC/DC systems is studied through the tool presented in [7], developed in MATPOWER ${ }^{\circledR}$ and based on extending an standard AC OPF with the constraints expressing the DC grid equations. For involving DC and AC power systems, the problem can be classified as non-linear constrained optimization. It is solved through an Interior Point Algorithm with barrier function [12].

The tool is applied to hybrid HVDC-HVAC systems integrating offshore wind power, whose general layout is sketched in Figure 1. The power produced by the wind power plants is injected in the DC grid through VSCs operating as rectifiers and it is transmitted to the AC grid through VSCs operating as inverters. The HVAC links enable the power transmission to the consumption nodes.

The available wind power in all the wind power plants, the active and reactive power demand, the electrical characteristics of the DC and AC grids, as well as the distances of lines and cables and the converter loss parameters are known data. The tool determines the active and reactive power injections from all the generating units and the power flowing through each branch that meet the demand with minimum power generation costs while ensuring the secure operation of the system.

\subsection{Contingencies and security constraints}

The contingencies considered in this study are the outage of any power system component: AC branch, AC generator, DC branch or VSC. The control possibilities of the TSO to face a disturbance depend on the nature of the contingency and on the time available to ensure that the system returns to a secure state.
Preventive actions or measures are taken before the contingency occurs and they are based on the fact that the TSO relies on the topology of the grid and on the appropriate selection of the generator and converter set-points to achieve a healthy state in case a contingency occurs. Meshing and redundant paths are examples of preventive measures. Corrective or curative measures are taken immediately after the incident happens and it is assumed that the TSO has enough time to ensure the system recovers to a healthy state, relying on the fact that all generators and VSC that do not suffer the contingency will participate in the regulation.

The possibility to use corrective actions depends on the actual state of the system, with varying degree of renewable generation. The existing operational reserves on the power system do also influence the capability of reaching a secure state. For this reason, the system spinning reserves have been included in the formulation, as described in Section 2.4.

\subsection{Generation and demand modelling}

The generation units in this study are separated in wind power plants and the rest of generating units. In both cases, they are aggregated in bigger units. As a consequence of weather conditions variations, the wind speed changes over time and this leads to wind power fluctuations. So, variability must be taken into account when modelling this resource. Another characteristic of wind power is uncertainty, which limits its predictability. In this study, forecasting errors are not considered. In order to include a realistic wind power generation profile, real data from the Belgian TSO, Elia, representing the Belgian aggregated wind farms production, have been used and scaled. The wind farms are modelled as AC generating units whose maximum output power is the available wind power of the wind power plant and they are characterized by lower generating costs than the rest of the generating units, so their injection is prioritised. The other generating units are aggregated, as well as the demand. For the load values representing the demand, data from the Belgian system has also been used and scaled.

The generation costs are modelled according to a second order polynomial and the wind power generation cost is assumed to be proportional to the power produced [13]. As the authors from [13] propose, it is assumed that the purchase of wind power is modelled as a fixed tariff (for the scheduled wind power) according to the feed-in tariff regulation.

\subsection{Spinning reserves modelling}

The term spinning reserves is defined in literature in various ways. According to NREL (National Renewable Energy Laboratory), spinning reserve is the generation and responsive load that is on-line, that can begin responding immediately, and is fully responsive within 10 minutes. According to NERC (North American Electric Reliability Council), spinning reserves is the unloaded generation that is synchronized and ready to serve additional demand. Many other definitions can be found in literature $[14,15]$ and, as identified in [16], some of them do not agree on who provides spinning reserve (in the 


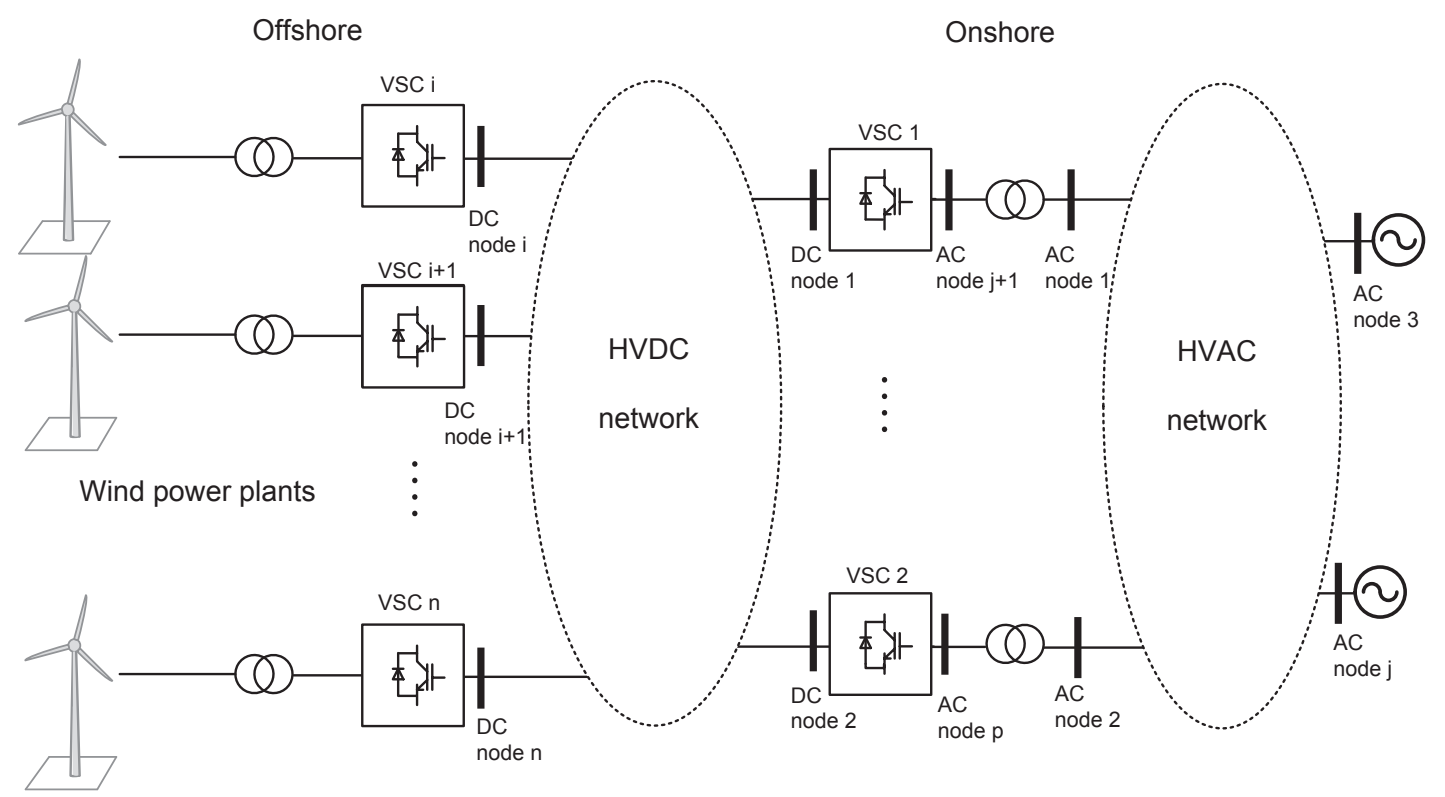

Fig. 1: Hybrid HVDC-HVAC system for integrating offshore wind power

sense that it is limited to generators or the demand can also participate), the time frame to respond to a request or how is this reserve activated (it happens automatically or it is only done at the request of the TSO). Thus, [16] proposes a general definition, assumed for this study: spinning reserve refers to the unused capacity which can be activated on decision of the system operator and which is provided by devices that are synchronized to the network and are able to affect the active power. Secondary control can be included in and tertiary control is considered spinning reserve.

Mainly two approaches exist for modelling spinning reserves. The first is deterministic and the second probabilistic [17]. However, some authors, like [18], propose a hybrid methodology. The main difference between the deterministic and probabilistic approach is that the latter includes the stochastic behaviour of system components by taking into account the load forecasting deviation, equipment failure and repair rates. For the sake of simplicity and for avoiding large computational times, the deterministic approach is adopted in this study. It is based on determining the spinning reserves for minimizing the operating cost up to a particular level of risk in the system throughout the operating period. The criteria for the total reserve estimation varies in each country. It can be one or a combination of fixed capacity margin, fixed percentage of system load, fixed percentage of on-line capacity or to fix it to be greater than the capacity of the largest on-line generating unit. For this study, the existing quantity of spinning reserves of the Belgian system have been scaled [17]. In the Belgian system the spinning reserves requirement criteria is currently at least $460 \mathrm{MW}$ by generators [16].

In this analysis, it is also assumed that demand will not contribute to spinning reserves, while wind power plants and the existing generating units will. While conventional generating units will have both ramping up and down capabilities, the wind power plants will only have ramping down capability. Wind power plants could have spinning reserve with ramping up capability if they would include a storage system, which is not considered in this study. The costs for reserves are modelled as direct costs, proportional to the amount of reserves provided by the generating unit. The variables and parameters listed in Subsections 2.4.1 and 2.4.2 allow the mathematical formulation of spinning reserves constraints, in Section 2.4.3.

\subsubsection{Variables}

- $i$ index of generating unit, $\mathrm{i}=1 . . n_{g}$, where $n_{g}$ is the number of generating units

- $P_{g i}$ active power generation from generating unit $i$

- $P_{d}$ corresponds to the total active power demand

- $R_{g i}^{u p}$ system spinning reserve up from generating unit $i$

- $R_{\text {gi }}^{\text {down }}$ system spinning reserve down from generating unit

\subsubsection{Parameters}

- $P_{g i}^{\min }$ minimum active power generation from generating unit $i$

- $P_{g i}^{\max }$ maximum active power generation from generating unit $i$

- $R M P^{u p}$ maximum ramp up rate of unit $i$

- $R M P^{\text {down }}$ maximum ramp down rate of unit $i$ 
- $\tau^{u p}$ is the time for ramping up. It corresponds to the time period considered (1h)

- $\tau^{\text {down }}$ is the time for ramping down. It corresponds to the time period considered (1h)

- $R^{u p}$ total system spinning reserve up, according to a deterministic criteria

- $R^{\text {down }}$ total system spinning reserve down, according to a deterministic criteria

\subsubsection{Constraints}

Equation (1) expresses the balance between generation and demand and Equations (2)-(3) reflect that the total spinning reserve up and down must fit the system spinning reserves needs.

$$
\begin{array}{r}
\sum_{i=1}^{n_{g}} P_{g i}=P_{d} \\
\sum_{i=1}^{n_{g}} R_{g i}^{u p} \geq R^{u p} \\
\sum_{i=1}^{n_{g}} R_{g i}^{\text {down }} \geq R^{\text {down }}
\end{array}
$$

Active power generation in each unit is limited, as shown in Equation (4).

$$
P_{g i}^{\min } \leq P_{g i} \leq P_{g i}^{\max }
$$

The spinning reserves delivered by each generating unit are limited, according to Equations (5)-(6), and must satisfy the ramp up and down rates defined in Equations (7)-(8).

$$
\begin{array}{r}
R_{g i}^{u p-m i n} \leq R_{g i}^{u p} \leq R_{g i}^{u p-\max } \\
R_{g i}^{\text {down-min }} \leq R_{g i}^{\text {down }} \leq R_{g i}^{\text {down-max }} \\
R_{g i}^{u p-\max }=R M P_{g i}^{u p} \times \tau^{u p} \\
R_{g i}^{\text {down-max }}=R M P_{g i}^{\text {down }} \times \tau^{\text {down }}
\end{array}
$$

The active power delivered by each generating unit and its ramping up and down reserves are limited by the maximum and minimum power available in this unit, as shown in Equations (9)-(10) respectively.

$$
\begin{aligned}
P_{g i}+R_{g i}^{u p} & \leq P_{g i}^{\max } \\
P_{g i}-R_{g i}^{\text {down }} & \geq P_{g i}^{\text {min }}
\end{aligned}
$$

These constraints are added to the SCOPF formulation. So the tool output will include the spinning reserves from each generating unit ( $R_{g i}^{u p}$ and $R_{g i}^{d o w n}$ ) needed to satisfy the total spinning reserves of the system while ensuring the stable operation with minimum generation costs.

\section{Methodology for determining the cost of operation over one year}

The flowchart of the SCOPF tool process applied to determine the cost of operation of a power system over one year is shown in Figure 2. The blue text applies for the particular study case detailed in Section 4.1. First, the system under study needs to be defined, specifying the configuration and electrical characteristics. Then, the type of security analysis to be performed is detailed, defining all the possible states of the system based on the elements that can be unavailable and on the security level assumed for the power system. Next, an iterative computation process starts, taking into consideration the time horizon for computing the cost of operation. Assuming the cost needs to be determined for one year operation, with hourly samples of generation and demand, the next steps will be run 8760 times.

For a specific objective function, knowing the cost of operation of the generating units, the spinning reserves location, capacity and cost, as well as the ramping up/down capabilities of the generating units, several SCOPF are executed (one, per each possible scenario of the power system at the specified hour), taking into consideration the corrective actions of the TSO. For each SCOPF, the cost of operation and the wind power curtailed are calculated and results are stored.

Once all the SCOPF have been performed, the total cost of operating the hybrid HVAC-HVDC system over one year is computed considering the probability of occurrence of the different operating states. So as to compute the probability of each state, the failure rate of the different components and the downtime to repair are considered. For the probabilistic analysis, the following criteria apply:

- Each power system component has two possible states: on-line and off-line

- The probability of component $i$ of being off-line (or under failure) is $u_{i}$

- The probability of component $i$ of being on-line (healthy) is $a_{i}=1-u_{i}$

- The probability of finding the system working in state $s$ is equal to the product of the probabilities of the $k$ available elements and the probabilities of the $N-k$ out-of-service elements, being $N$ the total number of system components

So, the probability of the system of working under state $s$ can be expressed as:

$$
P_{s}=\prod_{i=1}^{N} a_{1} a_{2} \ldots a_{k} \times u_{1} u_{2} \ldots u_{N-k}
$$




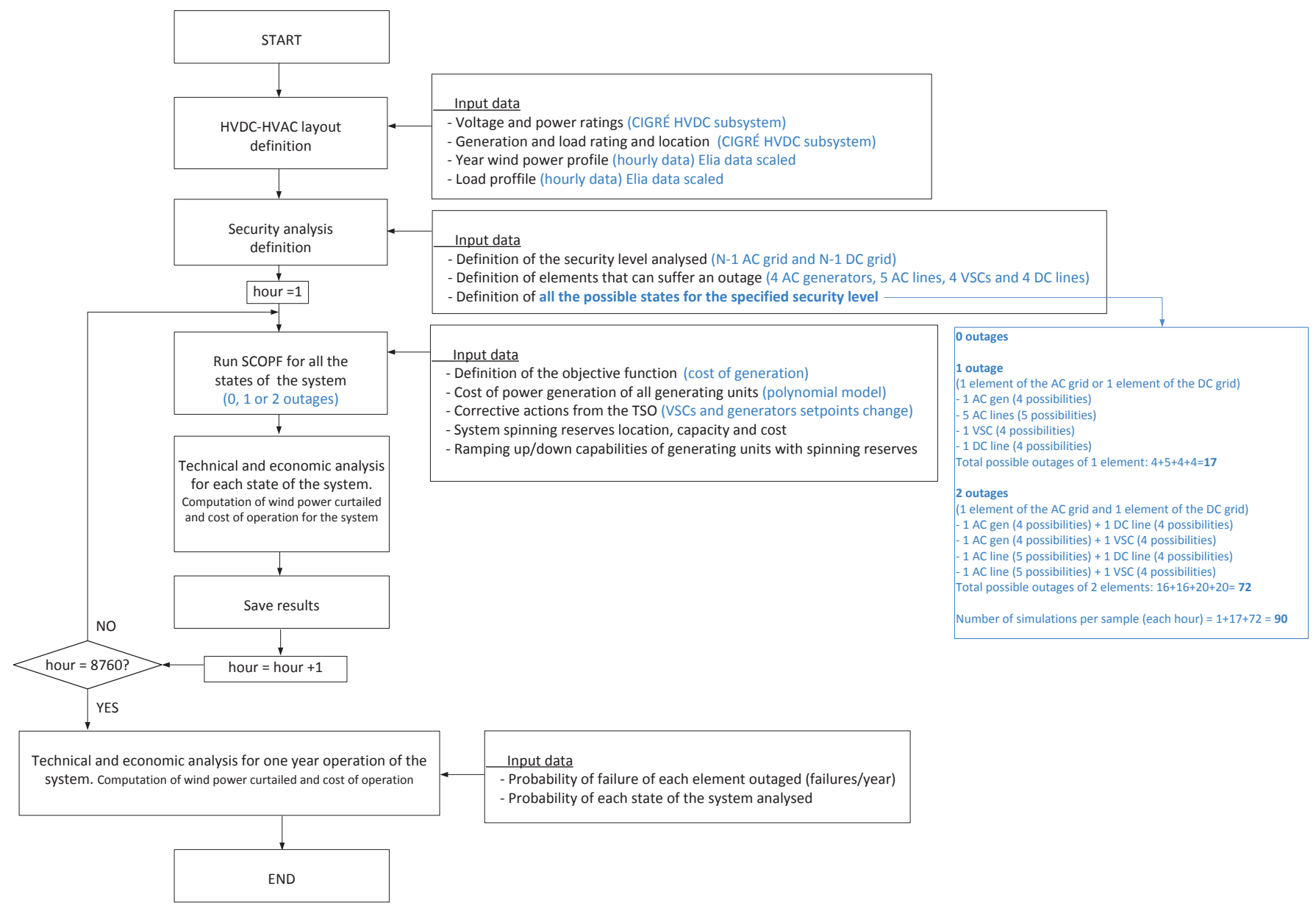

Fig. 2: Flowchart of the methodology

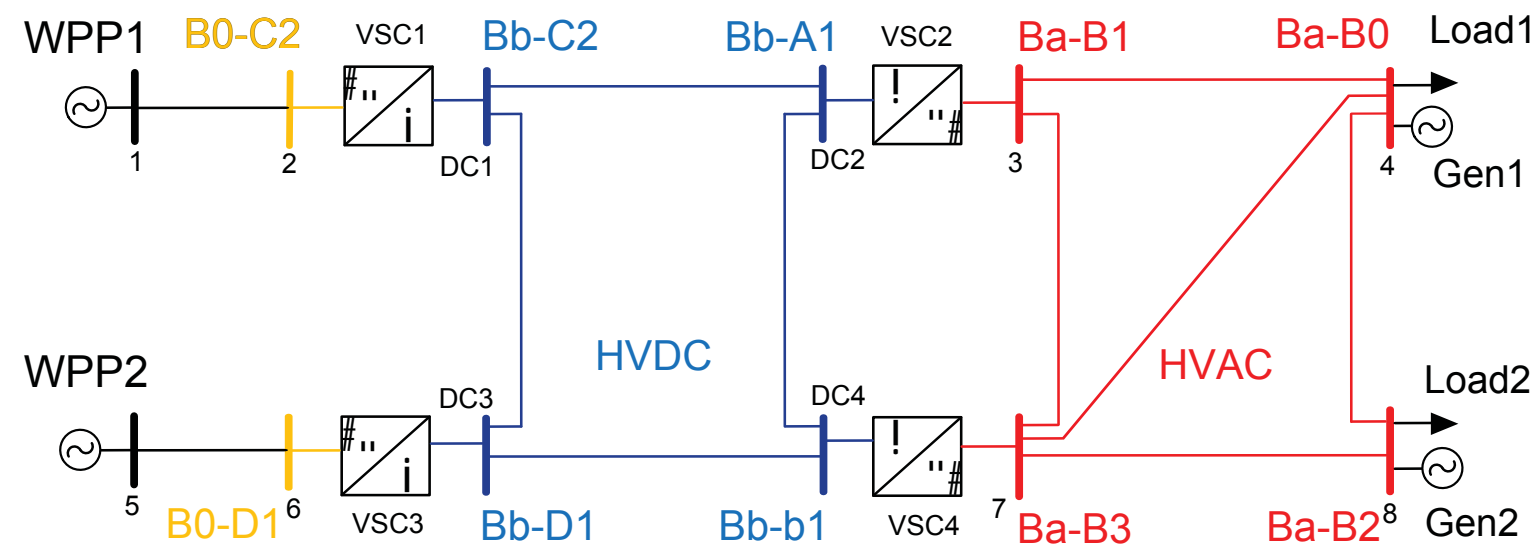

Fig. 3: HVDC-HVAC system based on [19] 


\section{Study case}

\subsection{System description}

The steps detailed in Section 3 are applied to a particular hybrid HVDC/HVAC system, inspired in the Test system defined by CIGRÉ in [19] and represented in Figure 3. The bus letters in Figure 3 correspond to the designation used in [19]. The AC system voltage is $380 \mathrm{kV}$ and the DC system voltage is $+/-400 \mathrm{kV}$. The wind farm VSCs are rated $800 \mathrm{MVA}$, while the grid side VSCs are rated 1200 MVA. The aggregated wind generation in Belgium in 2013 (taken from Elia [23]) is scaled and now grouped in 2 wind farms. So, the two wind farms present different wind proffiles (with correlated wind speeds). Similarly, the demand data for this country in 2013 (taken from Elia [23]) is scaled and grouped in 2 loads, with a similar share (random values in the range of $40-60 \%$ ). The generation that does not come form the wind resource is represented through two generating units connected to the same buses that the loads representing the demand.

The probability of unavailability of the different power system components is computed taking into consideration its failure rate (failures/year) and its downtime to repair (expressed in hours), from [20,24]. Their product gives the unavailability in hours/year, which can be turned into a probability by dividing it by the 8760 hours of a year. The failure rates of some power system elements can be found in the literature expressed as number of failures/year or number of failures/(100 km $\times$ year) for lines. The power system under study is constituted by 4 types of elements: AC branches, AC generators, DC branches and VSCs. Each element has a probability of failure. Furthermore, the probability of failure of lines depends on its length (so the probability of failure of the DC branches is different; but the same for all the AC branches because they have the same length), as reflected in Table 1. Based on the failure rates from [20], we set the failure rates per year in 0.006 failures/100 km for AC lines, 0.003 failures/100 km for DC bipolar lines and 1.4 failures/year for VSCs. The availability assumed for wind power plants and conventional power plants is extracted from $[24,25]$. Knowing the probability of failure for the different components and applying the probabilistic criteria described in Section 3, the probability of the system of working under normal operation is 0.7850 , the probability of losing 1 element (AC or DC) is 0.2081 (0.0289 for a DC element and 0.1791 for a $\mathrm{AC}$ element) and the probability of losing 2 elements (1 AC element and 1 DC element) is 0.0051. The system operates normally or with 1 or 2 elements under outage $99.82 \%$ of the time. The rest of the time corresponds to the sum of the probabilities of the scenarios not considered (outages of 3 up to 18 elements) and which can be neglected due to the low incidence they present in the total cost.

\subsection{Results}

The input data for the tool applied to the system described in Section 4.1 is plotted in Figures 4-5. It corresponds to the wind

\begin{tabular}{|c|c|c|}
\hline Branch & Failures/year & Repair time $(\mathrm{h})$ \\
\hline AC 3-4 (200 km) & 0.012 & 24 \\
AC 3-7 (200 km) & 0.012 & 24 \\
AC 4-7 (200 km) & 0.012 & 24 \\
AC 4-8 (200 km) & 0.012 & 24 \\
AC 7-8 (200 km) & 0.012 & 24 \\
DC 1-3 (400 km) & 0.012 & 1440 \\
DC 3-4 (400 km) & 0.012 & 1440 \\
DC 2-4 (300 km) & 0.009 & 1440 \\
DC 1-2 (200 km) & 0.006 & 1440 \\
\hline
\end{tabular}

Table 1: Failure rates and repair time of the system lines and cables [20]

power available in WPP1 plant and to the active power demand of Load 1. WPP2 and Load 2 have the same kind of inputs, but with different values. Although the months should have data for all their days, some months present one less day data due to the unavailability on the database consulted. The reactive power demand required by each load is assumed to be $10 \%$ of the active power demand it consumes.

For each hourly sample, a SCOPF is executed for each possible outage occurring. All the possible scenarios considered for each hour are represented in Figure 6. They correspond to normal operation, the loss of $1 \mathrm{AC}$ element, the loss of 1 DC element and the loss of 2 elements (1 AC and 1 DC). The cost corresponds to one hour evaluation of one day of january (without weighting it with the probability of occurrence of each state). It can be observed, that the most critical operation of the system appears when a VSC is lost or a VSC and a generator are lost.

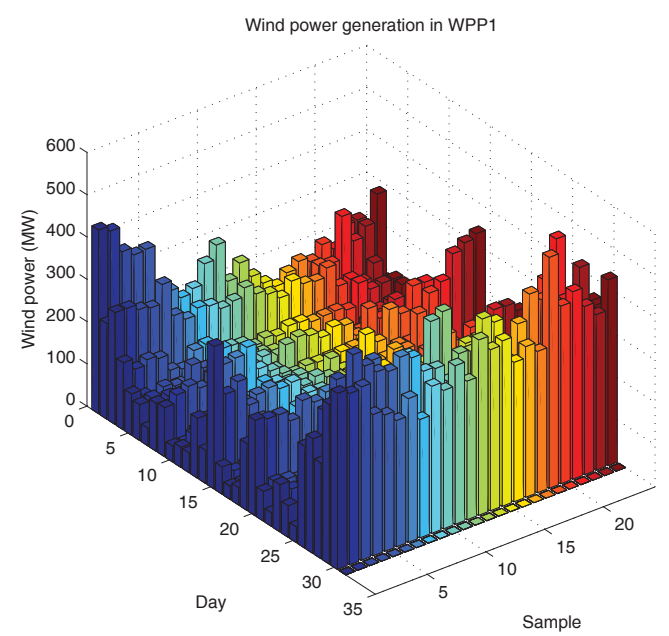

Fig. 4: Available wind power in WPP1

The cost of operation of the system, weighting the cost contribution of each scenario with its probability of occurrence, 


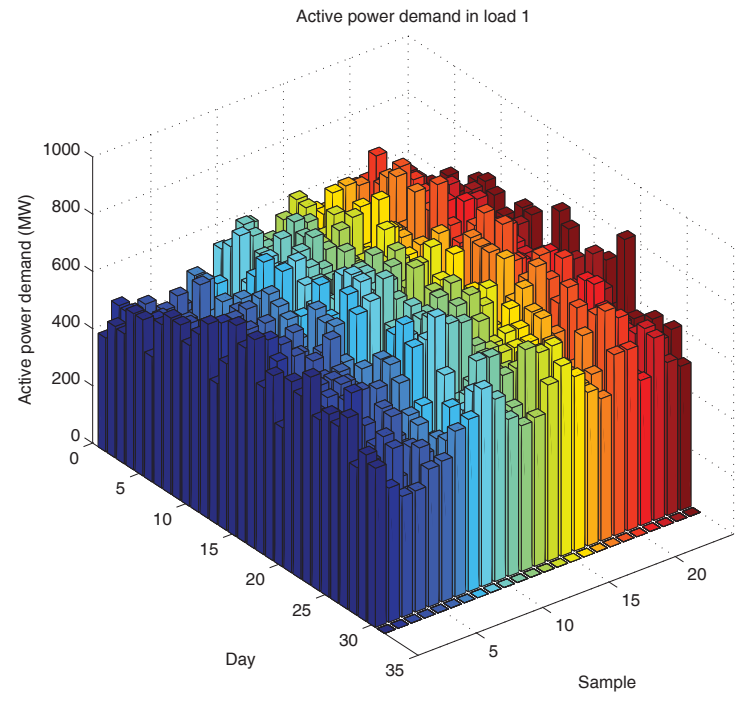

Fig. 5: Active power demand in Load 1

is represented monthly in Figure 7 for different security levels: normal operation, operation of the $\mathrm{AC}$ grid $\mathrm{N}-1$ and $\mathrm{DC}$ grid $\mathrm{N}-0$ (includes security against outages of $1 \mathrm{AC}$ element) and AC grid N-1 and DC grid N-1 (includes security against outages of $1 \mathrm{AC}$ or $1 \mathrm{DC}$ element and outages of $1 \mathrm{AC}$ and 1 DC element). Similarly, the wind power curtailment for the different security levels is shown monthly in Figure 8. The large wind power curtailment in the months of november and december is explained because while the total demand in the last months of the year increases around $17 \%$ compared to the month of july, the wind power available in november and december is more than double the existing on july. The growth in demand does not compensate for the significant increase of wind power, so more wind power curtailment is needed. The base case (normal operation) presents the lowest cost.

The total cost of operation and wind power curtailed during one year for the different security levels analysed are shown in Table 2 and Table 3. A higher security level of operation of the system leads to higher costs. The wind power needed to be curtailed also increases with the level of security as the power system becomes more constrained and can not absorb all the power injected as more elements are under outage and less power paths are available. Analysing the results obtained for the operation of AC grid N-1 and DC grid N-0 versus AC grid N-1 and DC grid N-1, it can be concluded that operating the DC grid N-1 implies an increase in the operating costs of 1.71 (M€/year) compared to its operation N-0. These results can help on the decision process for transmission expansion planning of hybrid AC/DC systems as, together with the investments needed, allows to perform cost-benefit analysis.

\begin{tabular}{|c|c|}
\hline Security level & Cost of operation (M€/year) \\
\hline Normal operation & 36.34 \\
AC grid N-1 \& DC grid N-0 & 44.91 \\
AC grid N-1 \& DC grid N-1 & 46.62 \\
\hline
\end{tabular}

Table 2: Total cost of operation and wind power curtailed during one year

\begin{tabular}{|c|c|}
\hline Security level & Wind curtailment (GWh/year) \\
\hline Normal operation & 99.33 \\
AC grid N-1 \& DC grid N-0 & 180.15 \\
AC grid N-1 \& DC grid N-1 & 200.66 \\
\hline
\end{tabular}

Table 3: Total wind power curtailed during one year

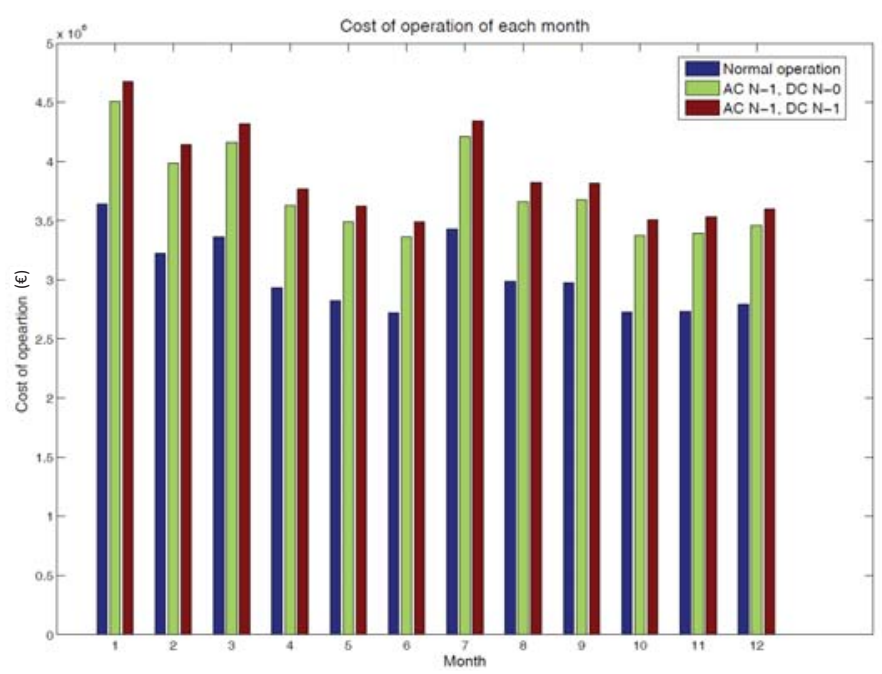

Fig. 7: Cost of operation for different security levels

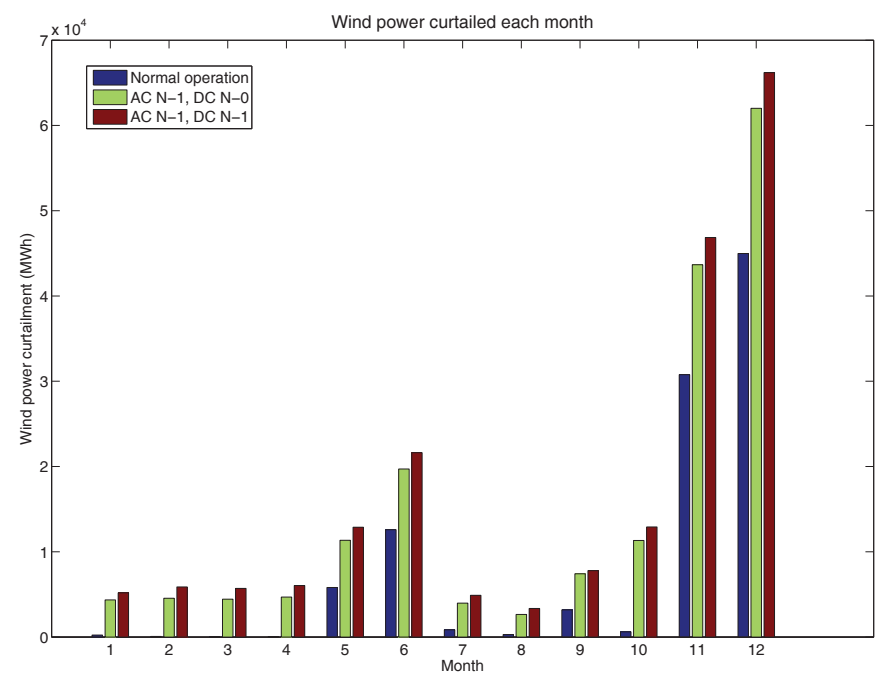

Fig. 8: Wind power curtailment for different security levels 

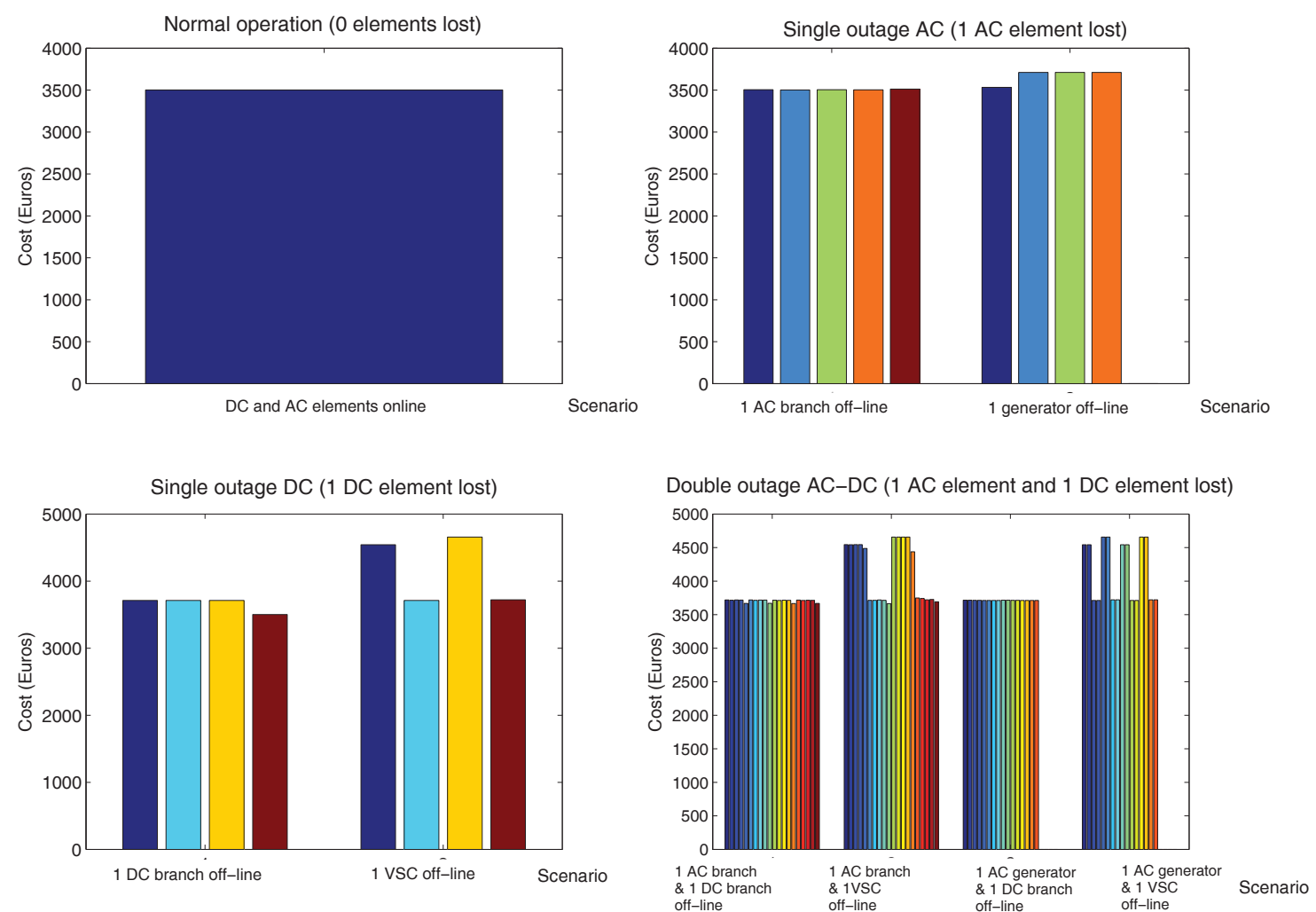

Fig. 6: Possible power system scenarios for each hour analysed

\section{Conclusions}

This study has proposed a methodology for evaluating the cost of operation over a year of a hybrid AC/DC system with high penetration of offshore wind under different security levels. A SCOPF tool has been used, modeling the TSO corrective measures for facing disturbances. The system studied has been operated so as to maximise the social welfare. The wind power variability is taken into consideration through real wind profiles. The system spinning reserves are included as additional constraints and the probability of the different scenarios is taken into account for the total cost computation. The application of this methodology to hybrid AC/DC systems allows to assess the value and the cost of operating the system with a higher or lower security. For the study case analysed, the operation of the DC grid N-1 leads to an increase in the costs of operation of 1 year of around 4\%, compared to the operation of the DC grid $\mathrm{N}-0$.

\section{Acknowledgements}

This work was supported by the Ministerio de Ciencia e Innovación under the projects ENE2012-33043 and ENE2013-47296 and by EIT KIC InnoEnergy project Smart Power. The research of Jef Beerten is funded by a postdoctoral fellowship from the Research Foundation - Flanders (FWO).

\section{References}

[1] P. Kundur. "Power System Stability and Control" Mc.Graw Hill, Inc, 1993.

[2] F. Capitanescu, J.L. Martinez Ramos, P. Panciatici, D. Kirschen, A. Marano Marcolini, L. Platbrood, and L. Wehenkel. "State-of-the-art, challenges, and future trends in security constrained optimal power flow", Electric Power Systems Research, 81(8):1731 - 1741, 2011.

[3] T.M. Haileselassie and K. Uhlen. "Power system security in a meshed north sea hvdc grid", Proceedings of the IEEE, 101(4):978-990, April 2013.

[4] Mohamadreza Baradar. "Modeling of multi terminal HVDC systems in power flow and optimal power flow formulations", KTH, 2013.

[5] Jun Cao, Wenjuan Du, H.F. Wang, and S.Q. Bu. "Minimization of transmission loss in meshed ac/dc grids with vsc-mtdc networks", Power Systems, IEEE Transactions on, 28(3):3047-3055, Aug 2013.

[6] R. Wiget and G. Andersson. "Optimal power flow for combined ac and multi-terminal hvdc grids", based on vsc converters. In Power and Energy Society General Meeting, 2012 IEEE, pages 1-8, July 2012. 
[7] J. Rimez. "Optimal operation of hybrid AC/DC meshed grids", PhD thesis, KU Leuven, Department of Electrical Engineering, 2014.

[8] Chatzivasileiadis S., Krause T., and Andersson G. "Security-constrained optimal power fl ow including postcontingency control of vsc hvdc lines", In 12 SEPOPE, Rio de Janeiro, pages 1-8, May 2012.

[9] M. Vrakopoulou, K. Margellos, J. Lygeros, and G. Andersson. Probabilistic guarantees for the $n-1$ security of systems with wind power generation. In Proceedings of PMAPS, Istanbul, June 2012.

[10] M. Vrakopoulou, K. Margellos, J. Lygeros, and G. Andersson. A probabilistic framework for security constrained reserve scheduling of networks with wind power generation. In Energy Conference and Exhibition (ENERGYCON), 2012 IEEE International, pages 452-457, Sept 2012.

[11] M. Vrakopoulou, S. Chatzivasileiadis, E. Iggland, M. Imhof, T. Krause, O. Makela, J.L. Mathieu, L. Roald, R. Wiget, and G. Andersson. A unified analysis of securityconstrained opf formulations considering uncertainty, risk, and controllability in single and multi-area systems. In Bulk Power System Dynamics and Control - IX Optimization, Security and Control of the Emerging Power Grid (IREP), 2013 IREP Symposium, pages 1-19, Aug 2013.

[12] Richard H. Byrd and Jean Charles Gilbert. A trust region method based on interior point techniques for nonlinear programming. Mathematical Programming, 89:149-185, 1996.

[13] S. Mishra, Y. Mishra, and S. Vignesh. 'Security constrained economic dispatch considering wind energy" conversion systems. In Power and Energy Society General Meeting, 2011 IEEE, pages 1-8, July 2011.

[14] Allen J Wood and Bruce F Wollenberg. Institution of Electrical Engineers, 2nd edition.

[15] G. Jordan J. Zhu and S. Ihara. "The mark et for spinning reserve and its impacts on energy prices" In Proceedings of the IEEE Power Engineering Society Winter Meeting, 2000.

[16] Daniel Kirschen Yann Rebours. "What is spinning reserve?" The University of Manchester.

[17] Ifedi Kenneth Odinakaeze. "Assessment of spinning reserve requirements in a deregulated system" 2010.

[18] F. Bouffard and F.D. Galiana."An electricity market with a probabilistic spinning reserve criterion" Power Systems, IEEE Transactions on, 19(1):300-307, Feb 2004.

[19] CIGRÉ Working Groups B4-58 ("Devices for load flow control and methodologies for direct voltage control in a meshed HVDC grid) and B4-57 (Guide for the development of models for HVDC converters in a HVDC grid"). The CIGRÉ B4 DC grid test system. International Council for Large Electric Systems (CIGRÉ), 2013.

[20] K. Lindén, B. Jacobson, M. Bollen, and J. Lundquist. "Reliability study methodology for hvdc grids" In B4-108 CIGRE, 2010.

[21] C. Hudon, M. Levesque, D. Nguyen, C. Millet, and F. Truchon. "Root cause analysis of generator failures" In Electrical Insulation (ISEI), Conference Record of the 2012 IEEE International Symposium on, pages 199-203, June 2012.

[22] Shuangwen (Shawn) Sheng. "Report on Wind Turbine Subsystem Reliability. A Survey of Various Databases" In NREL, 2013.

[23] Elia, Belgium electricity transmission system operator, http://www.elia.be/en/grid-data/

[24] Ole Holmstrom, Work Package 9: Electrical grid "Survey of reliability of large offshore wind farms " 2007 , UPWIND.

[25] H. C. Wels, "Generic ageing characteristics of conventional power plants, Lessons learned from data-analysis a model for life extension planning" 2007. 